\title{
Measurement of pulse wave velocity in children and young adults: a comparative study using three different devices
}

\author{
Eva Kis ${ }^{1}$, Orsolya Cseprekál ${ }^{1}$, Andrea Kerti ${ }^{1}$, Paolo Salvi ${ }^{2,3}$, Athanase Benetos ${ }^{2}$, Andras Tisler ${ }^{4}$, Attila Szabó ${ }^{1}$, \\ Tivadar Tulassay ${ }^{1}$ and György S Reusz ${ }^{1}$
}

To estimate the value of pulse wave velocity (PWV) in pediatric cardiovascular disease, prospective studies are needed. Various instruments based on different measurement principles are proposed for use in children, hence the need to test the comparability of these devices in this younger population. The objective of this study was to compare PWV measured by oscillometry (Vicorder (VIC)) with the gold standard of applanation tonometry (PulsePen (PP), Sphygmocor (SC)). PWV was measured in 98 children and young adults (age: 16.7(6.3-26.6) years (median(range)) with the above three devices at the same visit under standardized conditions. Mean PWV measured by VIC was significantly lower than that measured by SC and PP. There was no difference following path length correction of the VIC measurement (using the distance between the jugular notch and the center of the femoral cuff), (PP: 6.12(1.00), SC: 5.94(0.91), VIC: 6.14(0.75) $\mathrm{m} \mathrm{s}^{-1}$ ). Velocities measured by the three devices showed highly significant correlations. Bland-Altman analysis revealed excellent concordance between all three devices, however, there was a small but significant proportional error in the VIC measurements showing a trend toward lower PWV measured by VIC at higher PWV values. Our study provides data on the three most frequently used instruments in pediatrics. Following path length correction of the VIC, all three devices provided comparable results. Thus, our work allows extrapolating data between previously established normal PWV values for children and forthcoming studies using these instruments to assess children at long-term risk of cardiovascular disease. The small proportional error of VIC needs additional technical development to improve the accuracy of the measurements.

Hypertension Research (2011) 34, 1197-1202; doi:10.1038/hr.2011.103; published online 28 July 2011

Keywords: applanation tonometry; arterial stiffness; children; oscillometry; pulse wave velocity

\section{INTRODUCTION}

Cardiovascular disease is among the leading causes of death in Western societies. ${ }^{1}$ Deterioration in endothelial function and arterial stiffness are early events in the development of cardiovascular damage. Although there is ample evidence that arteriosclerosis begins in childhood, ${ }^{2-4}$ hard end points, such as stroke, ischemic heart disease and death, are rare or virtually lacking in the pediatric population. Thus, there is an increasing need to establish validated noninvasive predictors to forecast early arterial disease and to be able to characterize elevated cardiovascular risk in youngsters. ${ }^{5}$ Development of validated methods for noninvasive measurement of early atherosclerotic disease has the potential to change the paradigm for evaluation and treatment of elevated cardiovascular risk in youth by focusing on target-organ damage. ${ }^{5}$

In adults, several noninvasive attributes of atherosclerosis have become established as valid and reliable tools for refining cardiovascular risk in order to target individuals who need early intervention. ${ }^{5}$
In this context, it has been widely recognized that aortic pulse wave velocity (aPWV) is a sensitive marker of arterial stiffness and, consequently, of cardiovascular outcome. ${ }^{6-9}$

In a previous work, including more than 1000 children and teenagers, we provided reference values for pulse wave velocity (PWV), thereby constituting a suitable tool for longitudinal clinical studies assessing subgroups of children who are at long-term risk of cardiovascular disease. ${ }^{10}$

A multitude of various methods and techniques have been used to assess PWV in adults such as applanation tonometry, ${ }^{11,12}$ ultrasound, ${ }^{13,14}$ methods using mechanotransducers ${ }^{15}$ and computerized oscillometry; ${ }^{16}$ furthermore, a number of comparative studies with diverging results have been published concerning the comparability of the different methods. ${ }^{17-21}$

Instruments based on the principle of applanation tonometry (PulsePen (PP) (DiaTecne, Milan, Italy) and Sphygmocor (SC) (AtCor, Sydney, Australia) have been extensively used. They are easily 
applied in adults and children and have a fair reproducibility. ${ }^{22-26} \mathrm{PP}$ and SC devices have been validated invasively in adults. ${ }^{12,27}$

It is claimed that, in children, PWV measurements by applanation tonometry may be difficult to carry out as they are time consuming and require some degree of patient cooperation. ${ }^{9}$ Thus, manufacturers are promoting new easy-to-use instruments to enter the market as valid tools for measuring aPWV. The Vicorder (VIC) (Skidmore, Bristol, UK) is such a device based on the oscillometric technique to measure aPWV. It is presented as a method with little dependency on operator skills and experience (http://www.smt-medical. com/public).

\section{Aims}

The aim of the study was to test the comparability in children and young adults of VIC aPWV measurements with the reference standard of applanation tonometry, according to the guidelines for validation of noninvasive hemodynamic measurement devices. ${ }^{28,29}$

In a broader approach, our aim was to standardize the expression of PWV measured by different devices as suggested by the AHA guidelines. ${ }^{5}$

\section{METHODS}

\section{Patients}

Aortic PWV was measured in 98 children and young adults (39 male and 59 female). Hospital in-patients and healthy young adult volunteers were included in the study (33 healthy volunteers, 24 renal patients, 21 patients with minor neurological problems (headache), 12 diabetic patients and 8 patients with eating disorders). Anthropometric and medical data of the participants are: age: 16.7 (6.3-26.6) years (median, (range)), height: $166.5(105-188) \mathrm{cm}$, weight: 57 (15-92) kg, systolic blood pressure: $110(83-138) \mathrm{mm} \mathrm{Hg}$, diastolic blood pressure: $67(50-88) \mathrm{Hg}$ mms and heart rate: 60 (51-106) beats per min.

The study protocol was approved by the local ethics committee and informed consent was obtained from study participants or parents.

\section{Methods}

Three devices, PP, SC and VIC, were used for aPWV measurement at the same visit. To assure patient collaboration, all subjects of the study were made familiar with the devices and the test procedure before any study measurements were made. At least one set of measurements was performed before the start of the formal data collection to minimize a 'surprise reaction'.

The order of the measurements with the three devices was randomly chosen after $15 \mathrm{~min}$ in resting supine position in a quiet room (standardized conditions to afford hemodynamic stability). The results of two successful measurements with a given device were used in the calculations.

\section{Devices based on applanation tonometry}

The principle of measurement of the PP and SC devices is similar. The probe of the device is connected to an electrocardiogram unit while pressure and electrocardiographic signals are transmitted to a computer. Carotid-femoral or aortic PWV was measured by sequential recordings of the arterial pressure wave at the carotid and femoral arteries. Aortic PWV was defined as pulse wave travel distance divided by the time difference between the rise delay of the distal and proximal pulse according to the $\mathrm{R}$ wave belonging to the electrocardiogram qRs complex, and calculated by the software using the intersecting tangent algorithm. The pulse travel distance was defined as the difference between the distance from the carotid sampling site to the jugular notch and distance from the jugular notch to the femoral sampling site (Figure 1a) ${ }^{27}$ The pulse wave was calibrated by measuring brachial blood pressure immediately before each recording. The measurement of pulse pressure was discarded and repeated if blood pressure and heart rate varied by more than $10 \%$ in the carotid and femoral sites. Recordings were also discarded when the variability between consecutive systolic or diastolic waveforms was $>10 \%$ or when the amplitude of the pulse wave signal was $<80 \mathrm{mV} .^{10}$

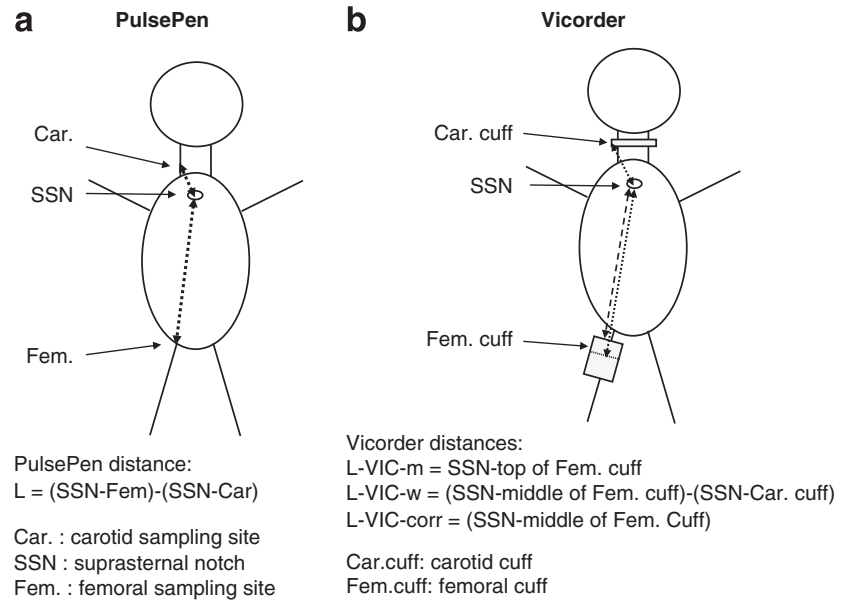

Figure 1 Graphical representation of the distances used by PulsePen/ Sphygmocor and Vicorder.

\section{PWV measured by oscillometry}

VIC simultaneously records the pulse wave from the carotid and femoral site by using the oscillometric method. Recording is achieved by first placing a neck pad, which is only inflatable over several centimeters, around the patient's neck. This pressure pad is applied over the right carotid artery to prevent compression of the trachea and compression of both carotid arteries at the same time. Next, a cuff is placed around the patient's right upper thigh to measure femoral pulse pressure. ${ }^{18} \mathrm{PWV}$ was measured by inflating the cuffs to $60 \mathrm{~mm} \mathrm{Hg}$ after which high-quality waveforms were recorded simultaneously using a volume displacement method. The foot-to-foot transit time was determined using a built-in cross-correlation algorithm centered on the peak of the second derivative of pressure. The signal of at least five heart cycles was used for a single determination of PWV.

Path length was defined in three ways: first, as the direct distance from the jugular notch to the top of the femoral cuff, as suggested by the manufacturer (L-VIC-m) (Vicorder User Manual Version 1.3.); ${ }^{18,30}$ second, as the difference between the distance from the jugular notch to the femoral sampling site and distance from the carotid sampling site to the jugular notch as suggested by Weber et al. (L-VIC-w); ${ }^{31}$ third, as the distance from the jugular notch to the femoral sampling site (L-VIC-corr) (Figure 1b).

To avoid possible interobserver variation, a single investigator (EK) carried out all PWV measurements. All measurements were performed at least twice to confirm reproducibility and the mean of the readings was used for further calculations. Femoral and carotid pulse pressure wave recordings were evaluated by a single observer (EK). The intraobserver coefficients of variations of PWV measurements were: PP 5.7\%, SC $7.2 \%$ and VIC 5.1\%.

\section{Statistical analysis}

Data analysis was performed using the STATISTICA 8.0 software (StatSoft Hungary Ltd., Budapest, Hungary). Data are presented as mean (s.d.) unless indicated otherwise. A ' $P$ ' value of $<0.05$ was considered as statistically significant.

Mean PWV values obtained by the different devices were compared by analysis of variance. Means were compared in post-hoc analysis by Tukey's multiple comparison test.

Linear regression analysis was performed to assess the correlation between the individual measurements obtained by the different devices. Both $\mathrm{R}$ coefficients and $P$ values are presented. In addition, the differences between the three devices were analyzed according to the Bland-Altman method. In this analysis, the difference between each pair of measurement was plotted against the mean of the pair and the three devices were compared pair by pair. The averages of two individual successful PWV results per patient were used.

The accuracy of the test device was determined both by the mean difference from the reference and the standard deviation of the difference (SDD) ${ }^{29}$ The accuracy was termed excellent, if the mean difference was $<0.5 \mathrm{~m} \mathrm{~s}^{-1}$ 
and SDD $<0.8 \mathrm{~m} \mathrm{~s}^{-1}$; acceptable, if the mean difference $<1.0 \mathrm{~m} \mathrm{~s}^{-1}$ and SDD $<1.5 \mathrm{~m} \mathrm{~s}^{-1}$; and poor, if the mean difference $<1.0 \mathrm{~m} \mathrm{~s}^{-1}$ or SDD $>1.5 \mathrm{~m} \mathrm{~s}^{-1} .^{29}$

\section{RESULTS}

Mean PWV values measured by the three instruments are: PP 6. 12 (1.00) $\mathrm{m} \mathrm{s}^{-1}$, SC $5.94(0.91) \mathrm{m} \mathrm{s}^{-1}$, VIC (L-m) 5.38 (0.72), VIC (L-w) $5.56(0.69) \mathrm{m} \mathrm{s}^{-1}$, VIC (L-corr) $6.14(0.75) \mathrm{m} \mathrm{s}^{-1}$. PWV by PP and SC did not differ significantly. PWV determined by VIC was significantly lower using the distances L-VIC-m and L-VIC-w than the PWV determined by SC and PP $(P<0.05)$. Following path length correction (L-VIC-corr), there was no difference in PWV measured by VIC compared with PP and SC.

PWV measured by the three devices showed highly significant correlations (Table 1).

Table 1 Correlations between PWV measured by PulsePen, Sphygmocor and Vicorder

\begin{tabular}{|c|c|c|}
\hline Correlation between PWV values $\left(m s^{-1}(s . d).\right)$ & r & $\mathrm{P}$ \\
\hline$P W V_{P P}-P W V_{S C}$ & 0.76 & $<0.0001$ \\
\hline 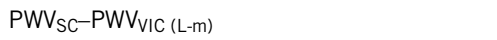 & 0.72 & $<0.0001$ \\
\hline 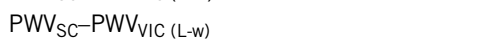 & 0.71 & $<0.0001$ \\
\hline$P W V_{S C}-P W V_{V I C}(L-c o r r)$ & 0.72 & $<0.0001$ \\
\hline 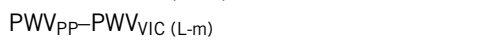 & 0.71 & $<0.0001$ \\
\hline 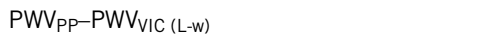 & 0.68 & $<0.0001$ \\
\hline 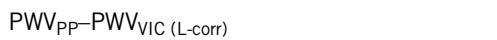 & 0.71 & $<0.0001$ \\
\hline
\end{tabular}

Abbreviations: L-corr, corrected path length for Vicorder (the distance from the jugular notch to the center of the femoral cuff); L-m, path length of Vicorder suggested by the manufacturer (the direct distance from the jugular notch to the upper edge of the femoral cuff); L-w, path length according to Weber et $a^{31}$ (the difference between the distance from the carotid sampling site to the jugular notch and distance from the jugular notch to the center of the femoral cuff) PP, PulsePen; PWV, pulse wave velocity; SC, Sphygmocor; VIC, Vicorder.

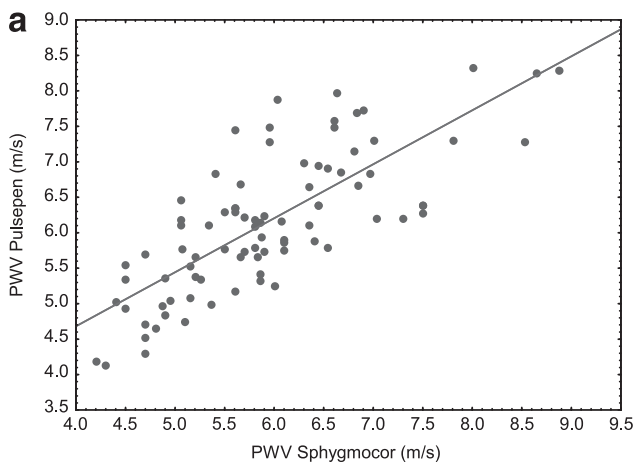

A graphical representation of the correlation between SC and PP is shown in Figure 2a.

A graphical representation of the correlation between the applanation tonometry-based devices and VIC is shown in Figure $2 \mathrm{~b}$ (PP and VIC (using path length L-VIC-corr)) and 2.c. (SC and VIC (using path length L-VIC-corr)).

The mean differences and SDDs according to the Bland-Altman analysis is shown in Table 2 .

The accordance between PP and SC was excellent. (Figure 3a)

Using the path length definition of the manufacturer, the mean difference between PP and VIC as well as that between SC and VIC was acceptable. Using the path length L-VIC-w, the mean difference between PP and VIC was acceptable whereas the mean difference between SC and VIC was excellent. Following path length correction (L-VIC-corr), these differences became excellent for both SC and PP devices (Table 2).

Graphical representations of the Bland-Altman plots between the applanation tonometry-based devices and VIC are shown in Figures $3 \mathrm{~b}$ (PP and VIC (using path length L-VIC-corr)) and $3 \mathrm{c}$ (SC and VIC (using path length L-VIC-corr)).

There was a slight but significant proportional bias on the Bland-Altman plots (Figures $3 \mathrm{~b}$ and $\mathrm{c}$ ), which could be expressed by the following equations:

For VIC and PP:

PWV $($ delta $)=-1.78+0.29 \times \mathrm{PWV}($ mean $) ; r=0.33, P=0.003$

For VIC and SC:

PWV $($ delta $)=-2.45+0.38 \times \mathrm{PWV}($ mean $) ; r=0.36, P=0.001$ where (delta) represents the mean of the differences between the PWV measured by PP or SC and VIC and (mean) represents the mean of PWV measured by PP or SC and VIC, respectively.

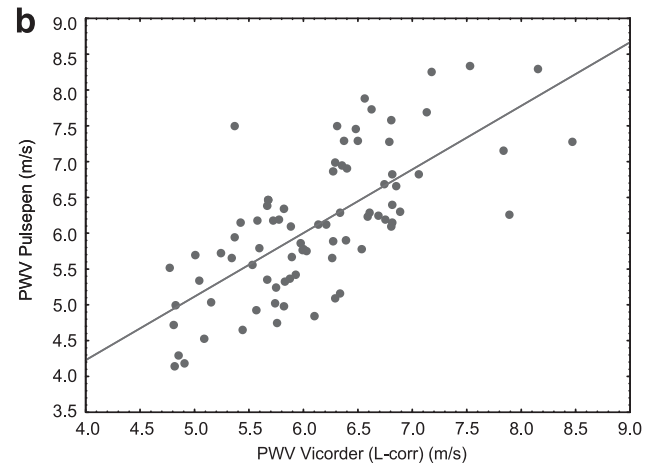

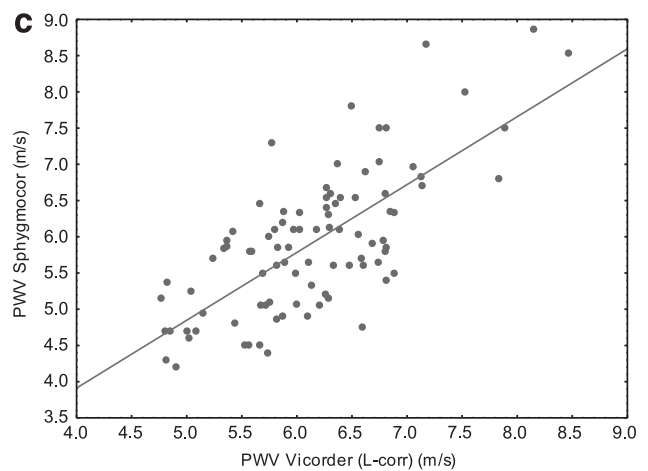

Figure 2 (a) Correlation between pulse wave velocity (PWV) measured by the Sphygmocor (SC) and the PulsePen (PP) device. $P W V_{\mathrm{PP}}=1.64+0.76 \times \mathrm{PWV}$ SC; $r=0.76, \quad P=0.0001$. (b) Correlation between PWV measured by the Vicorder (VIC) (with corrected path length) and the PP device.

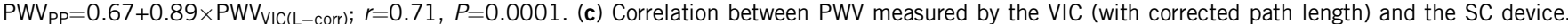
$\mathrm{PWV}_{\mathrm{SC}}=0.16+0.93 \times \mathrm{PWV} \mathrm{VIC}_{\mathrm{LL}-\mathrm{L} \text { (orr) }} ; r=0.72, P=0.0001$. A full color version of this figure is available at the Hypertension Research journal online. 
Table 2 Mean differences and standard deviation of the differences (SDD) of PWV neasured by the PulsePen, Sphygmocor and Vicorder devices according to the Bland-Altman analysis

\begin{tabular}{lcccr}
$\begin{array}{l}\text { Mean difference between } \\
\text { PWV values }\left(\mathrm{m} \mathrm{s}^{-1}(S D D)\right)\end{array}$ & PulsePen & Sphygmocor & Vicorder L-m & Vicorder L-w \\
\hline PulsePen & - & $0.12(0.77)$ & $0.73(0.71)$ & $0.53(0.73)$ \\
Sphygmocor & $-0.12(0.77)$ & - & $0.59(0.75)$ & $0.41(0.73)$
\end{tabular}

Abbreviations: L-corr, corrected path length for Vicorder (the distance from the jugular notch to the center of the femoral cuff); L-m, path length of Vicorder suggested by the manufacturer (the direct distance from the jugular notch to the upper edge of the femoral cuff); L-W, path length according to Weber et al. ${ }^{31}$ (the difference between the distance from the carotid sampling site to the jugular notch and distance from the jugular notch to the center of the femoral cuff); PWV, pulse wave velocity.

a
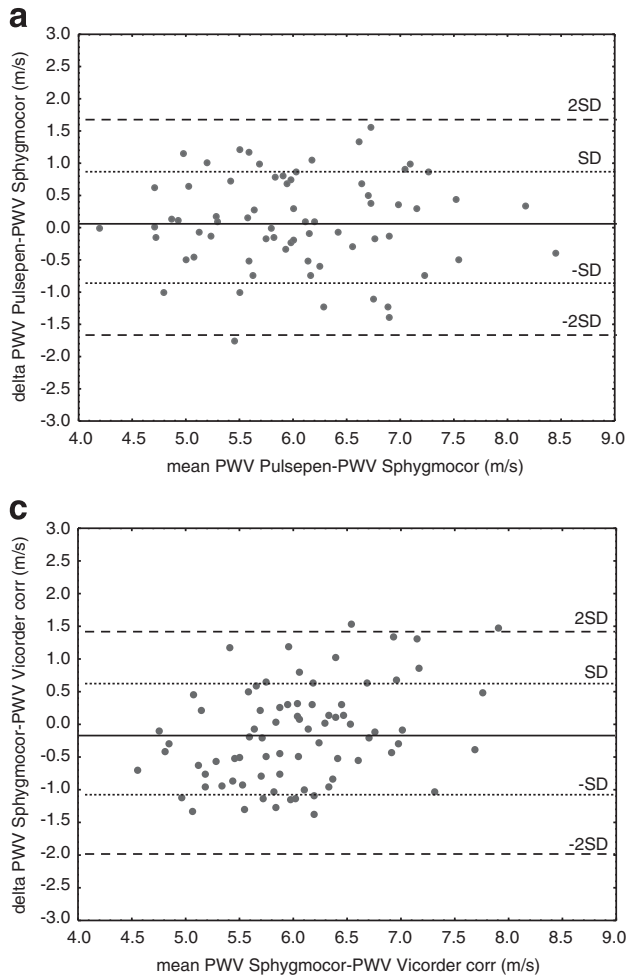

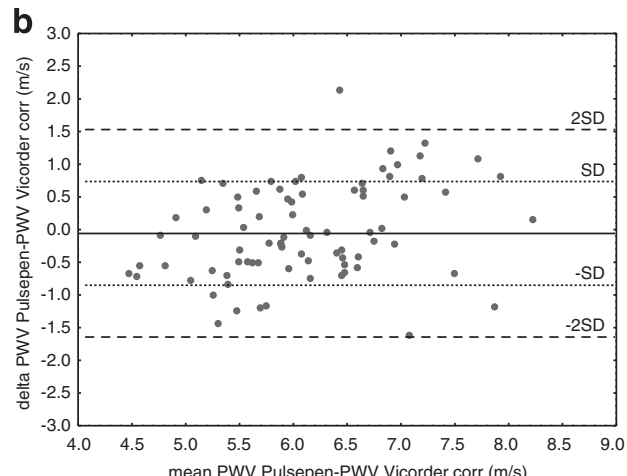

d

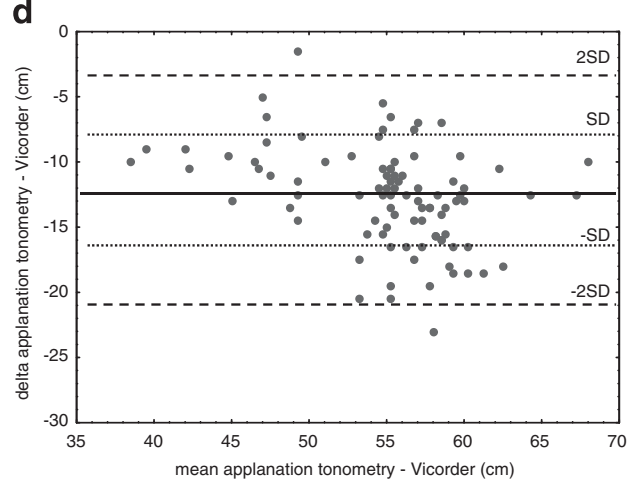

Figure 3 (a) Bland-Altman plots of pulse wave velocity (PWV) measured by Sphygmocor (SC) and PulsePen (PP). Delta PWV(PP)-PWV(SC): mean: $0.12 \mathrm{~m} \mathrm{~s}^{-1}$, s.d.: $0.77 \mathrm{~m} \mathrm{~s}^{-1}$. (b) Bland-Altman plots of PWV measured by Vicorder (VIC) (with corrected path length) and PP. Delta PWV(PP)-PWV(VIC(Lcorr)): mean: $-0.03 \mathrm{~m} \mathrm{~s}^{-1}$, s.d.: $0.72 \mathrm{~m} \mathrm{~s}^{-1}$. The equation describing the proportional error of the plot is PWV (delta) $=-1.78+0.29 \times P W V($ mean); $r=0.33$, $P=0.003$. (c) Bland-Altman plots of PWV measured by VIC (with corrected path length) and SC. Delta PWV(SC)-PWV(VIC(L-corr)): mean: $-0.17 \mathrm{~m} \mathrm{~s}^{-1}$, s.d.: $0.74 \mathrm{~m} \mathrm{~s}^{-1}$. The equation describing the proportional error of the plot is PWV (delta) $=-2.45+0.38 \times \mathrm{PWV}$ (mean); $r=0.36, P=0.001$. (d) Bland-Altman plots of path length used for the applanation tonometric devices and the corrected path length of VIC. Delta path length applanation tonometry-path length VIC corrected: mean: $-12.1 \mathrm{~cm}$, s.d.: $4.4 \mathrm{~cm}$. The equation describing the proportional error of the plot is Path length (delta) $=1.4-0.25 \times$ Path length (mean); $r=-0.36, P=0.0005$. A full color version of this figure is available at the Hypertension Research journal online.

To examine the possible cause of the proportional bias, we compared the transit times measured by the devices and the path length between the measuring probes using Bland-Altman analysis.

The mean difference and the SDDs between transit times of PP and SC was -3.8 (22.4) ms. The mean difference between PP and VIC was $-17(23.8) \mathrm{ms}$, and that between SC and VIC was -11.8 (11.7) ms. There was no significant proportional error on the Bland-Altman analysis.

The path length used for the PP and the SC was identical, thus no statistical analysis was performed.

The mean difference and the SDDs of the path length between the applanation tonometric devices and VIC (using path length L-VICcorr) was $-12.1(4.4 \mathrm{~cm})$.
There was a significant proportional bias on the Bland-Altman analysis (Figure $3 \mathrm{~d}$ ) that could be expressed by the following equation (using path length L-VIC-corr):

Path length $($ delta $)=1.4-0.25 \times$ path length $($ mean $) ; \quad r=-0.36$, $P=0.0005$

where (delta) represents the mean of the differences between the distances used for applanation tonometry and VIC (L-VIC-corr); (mean) represents the mean of these distances.

In order to assess whether the accordance between the devices is influenced by age, we performed a subgroup analysis of children younger than 18 years $(n=54$, mean age (s.d.): 13.34 (2.84) years) and compared it with the group of young adults aged $>18$ years $(22.73$ (2.46) years). 
The Bland-Altman analysis revealed an excellent concordance between the devices in both age groups (for VIC using path length L-VIC-corr).

Mean differences and SDDs of PWV measured are as follows:

For $P P$ and $S C$ :

-0.03 (0.78) (for the $<18$ group) vs. 0.29 (0.74) (for the $>18$

group); $\mathrm{p}=\mathrm{NS}$

For SC-VICcorr:

$-0.08(0.74)$ ) (for the $<18$ group) vs. $-0.27(0.74)$ (for the $>18$

group); $\mathrm{p}=\mathrm{NS}$

For PP-VICcorr:

-0.11 (0.75) (for the $<18$ group) vs. 0.06 (0.68) (for the $>18$ group); $\mathrm{p}=\mathrm{NS}$

Further, a similar proportional bias was observed in both age groups as described previously for the whole age group $(r=0.46$; $P=0.003$ for children aged $<18$ years and $r=0.41 ; P=0.007$ for young adults aged $>18$ years).

\section{DISCUSSION}

According to the American Heart Association's Recommendations for Noninvasive Assessment of Subclinical Atherosclerosis in Children and Adolescents, noninvasive methods for assessing cardiovascular risk in the pediatric population need to be standardized. ${ }^{5}$ Although studies based on standardization guidelines ${ }^{28,29}$ are not yet widely prevalent, they nevertheless present a valuable basis for further comparative studies. A number of comparative and validation studies have already been performed using different settings. ${ }^{19,21}$

The aim of the present work was to provide comparative values for PWV in children, teenagers and young adults across a broad spectrum of PWV using PP, SC and VIC devices adhering to or surpassing the pediatric portion of the guidelines proposed. ${ }^{29}$

The applanation tonometry devices $\mathrm{PP}$ and SC are invasively validated. ${ }^{12,27}$ Both use electrocardiogram-gated sequential tonometric measurements to asses aortic (carotid-femoral) PWV and are widely used in clinical and research practice. ${ }^{23-25}$ Survival studies in adults indicate that PWV measured by applanation tonometry has a good clinical predictive value. ${ }^{20}$

Involving more than 1000 children, our research group has furthermore confirmed the applicability of the PP device to mass screening of children of different age groups. ${ }^{10}$ The method proved to be fairly reproducible and provided reference values for PWV in children and teenagers. Thus, it remained of importance to compare and validate other devices to be potentially used in children. ${ }^{5,29}$

The newly developed VIC device is based on a different principle compared with PP and SC. It simultaneously records high-quality pressure waves by a volume displacement method. While still requiring strict settings regarding measurement conditions (quiet, thermoneutral environment, $15 \mathrm{~min}$ rest before measurements, and so on), the measurement itself, however, is claimed to be simple and not requiring the presence of a highly trained personnel.

Currently, PWV measured by VIC is not validated invasively and survival studies showing its predictive value are also lacking.

In the present study, pulse wave travel distance was determined in a similar manner for the instruments using applanation tonometry in order to avoid bias due to distance measurement.

Surface tape measurements were performed between the carotid sampling site and the jugular notch and between the jugular notch and the femoral sampling site. The difference between these two distances was considered to be the closest to the actual path length determined by invasive measurement as shown by Weber et al. ${ }^{31}$
The definition of the path length for the VIC device was more difficult. The original instruction manual proposes to measure the distance between the jugular notch and the upper end of the tight cuff directly (Vicorder User Manual. Version 1.3.). ${ }^{30}$ However, the femoral sampling site of VIC is by definition distal to that of SC and PP due to a different principle of measurement. In addition, according to Hickson et al, the actual detection point of VIC is $6.5 \mathrm{~cm}$ further distal to the upper end of the femoral cuff. Accordingly, in the most recent VIC manual, the femoral sampling site is defined as the center of the tight cuff (Vicorder User Manual. Version 1.3.2). ${ }^{30}$

Hence, VIC data were assessed herein using three measurements: first, the direct distance between the jugular notch and the top of the tight cuff; ${ }^{30}$ second, the difference between the distance from the carotid sampling site to the jugular notch and distance from the jugular notch to the femoral sampling site defined as the center of the femoral cuff ${ }^{18}$ - a path length analogous to that used by the PP and the SC instruments; ${ }^{31}$ third, the distance between the jugular notch and the center of the femoral cuff. $^{32}$ It is to note, that all three procedures are approximations and absolute differences are unimportant in intervention studies with repeated measures. However, when comparing two populations or pooling data for normal values or for meta-analyses, differences in the methods used to assess the path length will be critically important. ${ }^{9}$

The first major result of the present work is that the data obtained by the two devices using applanation tonometry are in excellent accordance with each other. This high concordance between PP and SC indicates that the established PWV reference values can be used for studies with both devices.

The VIC device, on the other hand, underestimated PWV in comparison with applanation tonometry using both the path length proposed by the manufacturer and that used by Weber et al. That occurred despite taking into account in the second definition of distance (L-VIC-w) that the actual detection point of the pulse wave by the VIC is at the center of the tight cuff. ${ }^{18}$

The best concordance with the applanation tonometric devices was achieved with the modified path length, a result in accordance with the findings of Mathieu et al., comparing surface tape measurements to data obtained by magnetic resonance imaging. ${ }^{32}$ Indeed, after correction of the distance, there was no longer any difference between the average values measured by the devices, and concordance was excellent with both SC and PP. The results were similar if patients younger than 18 years and those older than 18 years were assessed separately.

When comparing VIC and SC devices in adults, a proportional error due to the more distal detection point of VIC has been described by Hickson et al. ${ }^{18}$ They hypothesized that the inherent bias may be related to the fact that the VIC, but not the SC device, includes a longer segment of femoral artery. If the stiffness of this additional arterial segment is relatively constant between individuals, it may introduce an inherent bias at high aPWV. Indeed, the observation that peripheral PWV does not vary greatly throughout life supports this hypothesis.

Similarly, there was a small but significant proportional error in the VIC measurements in our patients showing a trend toward lower PWV measured by VIC at higher PWV values compared with the applanation tonometric instruments. The analysis of the transit times of the devices revealed no proportional bias (although per definition VIC had longer transit times than PP or SC due to the longer path length). This proportional error was rather due to the fact that the femoral sampling site of the VIC is distal to that of applanation tonometry (an average distance of $12.1(4.4) \mathrm{cm}$ for the path length of VIC in our study) and that distance is proportionally higher for longer total distances (that means taller patients). Based on the equations 
presented, the distortion is irrelevant at the average $\mathrm{PWV}$ value of $6 \mathrm{~m} \mathrm{~s}^{-1}\left(-0.03 \mathrm{~m} \mathrm{~s}^{-1}\right.$ for PP and $-0.17 \mathrm{~m} \mathrm{~s}^{-1}$ for SC).

In contrast, the present data are conflicting with the results of van Leeuwen-Segarceanu, who compared SC and VIC and found that the limit of agreement (defined as two times the SDDs between the two instruments per patient) was -3.50 to $4.66 \mathrm{~m} \mathrm{~s}^{-1} .21$ These authors referred to technical difficulties especially with the use of the carotid cuff as well as lack of experience or previous descriptions of the use of the VIC, which may have contributed to the results of their study. In the study herein, we did not meet specific difficulties with the VIC device such that the standard deviations of the differences between the devices compared were in a much narrower range $\left(0.71-0.75 \mathrm{~m} \mathrm{~s}^{-1}\right.$, see also Table 2).

In conclusion, after applying a path length adjustment for the VIC device, all instruments tested were in excellent concordance using the ARTERY Society guidelines. ${ }^{29}$ Thus, the average PWV measured in children by SC, PP and VIC all provided comparable results. The proportional error of VIC compared with PP and SC is due to the additional femoral path length. The equations presented could also be used when comparing the VIC data to the normal reference values of PWV established by applanations tonometry. ${ }^{10}$

\section{Perspective}

As PWV becomes more frequently measured in children and the number of commercially available devices is on the rise, it is important that common standards are agreed upon, so that devices are seen as interchangeable. Our study provides data on the two most frequently used instruments (PP and SC), and on VIC, a newly introduced device used in a recently initiated multicenter study. ${ }^{33}$ Following correction of path length of VIC, the three devices provided comparable results. Thus, our work allows extrapolating data between previously established normal PWV values for children and forthcoming studies using these instruments. However, the small proportional error of VIC needs additional analysis and technical development to improve the accuracy of the measurements.

\section{CONFLICT OF INTEREST}

Paolo Salvi is a consultant for PP (DiaTecne).

\section{ACKNOWLEDGEMENTS}

The study was supported by OTKA-071730, 100909, TÁMOP-4.2.2-08/1/KMR2008-0004, ETT 06-123/2009.

1 Najjar SS, Scuteri A, Lakatta EG. Arterial aging. Is it an immutable cardiovascular risk factor? Hypertension 2005; 46: 454-462.

2 Juonala M, Järvisalo J, Mäki-Torkko N, Kähönen M, Viiakari JSA, Raitakari OT. Risk factors identified in childhood and decreased carotid artery elasticity in adulthood: the cardiovascular risk in Young Finns Study. Circulation 2005; 112: 1486-1493.

3 Aggoun Y, Szezepanski I, Bonnet D. Noninvasive assessment of arterial stiffness and risk of atherosclerotic events in children. Pediatr Res 2005; 58: 173-178.

4 Li S, Chen W, Shrinivasan SR, Berenson GS. Childhood blood pressure as a predictor of arterial stiffness in young adults: the Bogalusa heart study. Hypertension 2004; 43: 514-546.

5 Urbina EM, Williams RV, Alpert BS, Collins RT, Daniels SR, Hayman L, Jacobson M, Mahoney L, Mietus-Snyder M, Rocchini A, Steinberger J, McCrindle B. American Heart Association Atherosclerosis, Hypertension and Obesity in Youth Committee of the council on cardiovascular disease in the young. Noninvasive assessment of subclinical atherosclerosis in children and adolescents: recommendations for standard assessment for clinical research: a scientific statement from the American Heart Association. Hypertension 2009; 54: 919-950.

6 Bramwell LC, Hill AV. Velocity of transmission of the pulse wave. Lancet 1922; 197: 891-892.

7 Khosdel AR, Carney SL, Nair BR, Gillies A. Better management of cardiovascular diseases by pulse wave velocity: combining clinical practice with clinical research using evidenced based medicine. Clin Med Res 2007; 5: 45-52.
8 Pannier B, Guerin AP, Marchais SJ, Safar ME, London GM. Stiffness of capacitive and conduit arteries: prognostic significance for end-stage renal disease patients. Hypertension 2005; 45: 592-596.

9 Laurent S, Cockcroft J, Van Bortel L, Boutouyrie P, Giannattasio C, Hayoz D, Pannier B, Vlachopoulos C, Wilkinson I, Struijker-Boudier H. European Network for Non-invasive Investigation of Large Arteries. Expert consensus document on arterial stiffness: methodological issues and clinical applications. Eur Heart J 2006; 27: 2588-2605.

10 Reusz GS, Cseprekal O, Temmar M, Kis E, Cherif AB, Thaleb A, Fekete A, Szabó AJ, Benetos $A$, Salvi $P$. Reference values of pulse wave velocity in healthy children and teenagers. Hypertension 2010; 56: 217-224.

11 McEniery CM, Yasmin, Hall IR, Qasem A, Wilkinson IB, Cockcroft JR. Normal vascular aging: differential effects on wave reflection and aortic pulse wave velocity: the Anglo-Cardiff Collaborative Trial (ACCT). J Am Coll Cardiol 2005; 46: 1753-1760.

12 Pauca AL, O'Rourke MF, Kon ND. Prospective evaluation of a method for estimating ascending aortic pressure from the radial artery pressure waveform. Hypertension 2001; 38: 932-937.

13 Cruickshank K, Riste L, Anderson SG, Wright JS, Dunn G, Gosling RG. Aortic pulse-wave velocity and its relationship to mortality in diabetes and glucose intolerance: an integrated index of vascular function? Circulation 2002; 106: 2085-2090.

14 Lehmann ED, Hopkins KD, Rawesh A, Joseph RC, Kongola K, Coppack SW, Gosling RG. Relation between number of cardiovascular risk factors/events and noninvasive Doppler ultrasound assessments of aortic compliance. Hypertension 1998; 32: 565-569.

15 Asmar R, Benetos A, Topouchian J, Laurent P, Pannier B, Brisac AM, Target R, Levy BI. Assessment of arterial distensibility by automatic pulse wave velocity measurement. Validation and clinical application studies. Hypertension 1995; 26: 485-490.

16 Baulmann J, Schillings U, Rickert S, Uen S, Dusing R, Illyes M, Cziraki A, Nickering G, Mengden T. A new oscillometric method for assessment of arterial stiffness: comparison with tonometric and piezo-electronic methods. J Hypertens 2008; 26: 523-528.

17 Salvi P, Magnani E, Valbusa F, Agnoletti D, Alecu C, Joly L, Benetos A. Comparative study of methodologies for pulse wave velocity estimation. J Hum Hypertens 2008; 22: 669-677.

18 Hickson SS, Butlin M, Broad J, Avolio AP, Wilkinson IB, McEniery CM. Validity and repeatability of the Vicorder apparatus: a comparison with the SphygmoCor device. Hypertens Res 2009; 32: 1079-1085.

19 Rajzer MW, Wojciechowska W, Klocek M, Palka I, Brzozowska-Kiszka M, KaweckaJaszcz K. Comparison of aortic pulse wave velocity measured by three techniques: Complior, SphygmoCor and Arteriograph. J Hypertens 2008; 26: 2001-2007.

20 Nemcsik J, Egresits J, El Hadj Othmane T, Fekete BC, Fodor E, Szabó T, Járai Z, Jekkel C, Kiss I, Tislér A. Validation of arteriograph - a new oscillometric device to measure arterial stiffness in patients on maintenance hemodialysis. Kidney Blood Press Res 2009; 32: 223-229.

21 van Leeuwen-Segarceanu EM, Tromp WF, Bos WJ, Vogels OJ, Groothoff JW, van der Lee $\mathrm{JH}$. Comparison of two instruments measuring carotid-femoral pulse wave velocity: Vicorder versus SphygmoCor. J Hypertens 2010; 28: 1687-1691.

22 Wilkinson IB, Fuchs SA, Jansen IM, Spratt JC, Murray GD, Cockcroft JR, Webb DJ. Reproducibility of pulse wave velocity and augmentation index measured by pulse wave analysis. J Hypertens 1998; 16: 2079-2084.

23 Frimodt-Moller M, Nielsen AH, Kamper AL, Strandgaard S. Reproducibility of pulse-wave analysis and pulse-wave velocity determination in chronic kidney disease. Nephrol Dial Transplant 2008; 23: 594-600.

24 Alecu C, Labat C, Kearney-Schwartz A, Fay R, Salvi P, Joly L, Lacolley P, Vespignani H, Benetos $A$. Reference values of aortic pulse wave velocity in the elderly. $J$ Hypertens 2008; 26: 2207-2212.

25 Kis E, Cseprekal O, Horvath Z, Katona G, Fekete BC, Hrapka E, Szabo A, Szabo AJ, Fekete A, Reusz GS. Pulse wave velocity in end-stage renal disease: influence of age and body dimensions. Pediatr Res 2008; 63: 95-98.

26 Cseprekal O, Kis E, Schaffer P, Othmane Tel H, Fekete BC, Vannay A, Szabo AJ, Remport A, Szabo A, Tulassay T, Reusz GS. Pulse wave velocity in children following renal transplantation. Nephrol Dial Transplant 2009; 24: 309-315.

27 Salvi P, Lio G, Labat C, Ricci E, Pannier B, Benetos A. Validation of a new noninvasive portable tonometer for determining arterial pressure wave and pulse wave velocity: the PulsePen device. J Hypertens 2004; 22: 2285-2293.

28 Van Bortel LM, Duprez D, Starmans-Kool MJ, Safar ME, Giannattasio C, Cockcroft J, Kaiser DR, Thuillez C. Clinical applications of arterial stiffness, Task Force III: recommendations for user procedures. Am J Hypertens 2002; 15: 445-452.

29 Wilkinson IB, McEniery CM, Schillaci G, Boutouyrie P, Segers P, Donald A, Chowienczyk PJ, On behalf of the ARTERY Society. ARTERY Society guidelines for validation of non-invasive haemodynamic measurement devices: part 1 , arterial pulse wave velocity. Artery Res 2010; 4: 34-40.

30 Vicorder User Manual. SMT Medical Technology GmbH, Würzburg, Germany, 2009 page. http://www.smt-medical.com/public.

31 Weber T, Ammer M, Rammer M, Adji A, O'Rourke MF, Wassertheurer S, Rosenkranz S, Eber B. Noninvasive determination of carotid-femoral pulse wave velocity depends critically on assessment of travel distance: a comparison with invasive measurement. J Hypertens 2009; 27: 1624-1630.

32 Mahieu D, Devos D, Kips J, Achten E, Segers P, Van Bortel L. Pulse wave velocity: how to assess the distance? Artery Res 2009; 3: 161 (abstract).

33 Querfeld U, Anarat A, Bayazit AK, Bakkaloglu AS, Bilginer Y, Caliskan S, Civilibal M, Doyon A, Duzova A, Kracht D, Litwin M, Melk A, Mir S, Sözeri B, Shroff R, Zeller R, Wühl E, Schaefer F, The 4C Study Group. The Cardiovascular Comorbidity in Children with Chronic Kidney Disease (4C) Study: objectives, design, and methodology. Clin J Am Soc Nephrol 2010; 5: 1642-1648. 